\title{
Microstructure Maps of Complex Perovskite Materials from Extensive Monte Carlo Sampling Using Machine Learning-Enabled Energy Model
}

Hsin-An Chen ${ }^{a}$, Ping-Han Tang ${ }^{a}$, Guan-Jie Chen $^{b}$, Chien-Cheng Chang ${ }^{b}$, and Chun-Wei Pao*a,c

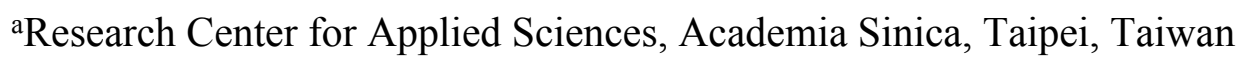

'Institute of Applied Mechanics, National Taiwan University, Taipei, Taiwan

'Department of Photonics, National Chiao Tung University, Hsin-Chu, Taiwan

AUTHOR INFORMATION

Corresponding Author

*E-mail: cwpao@gate.sinica.edu.tw 


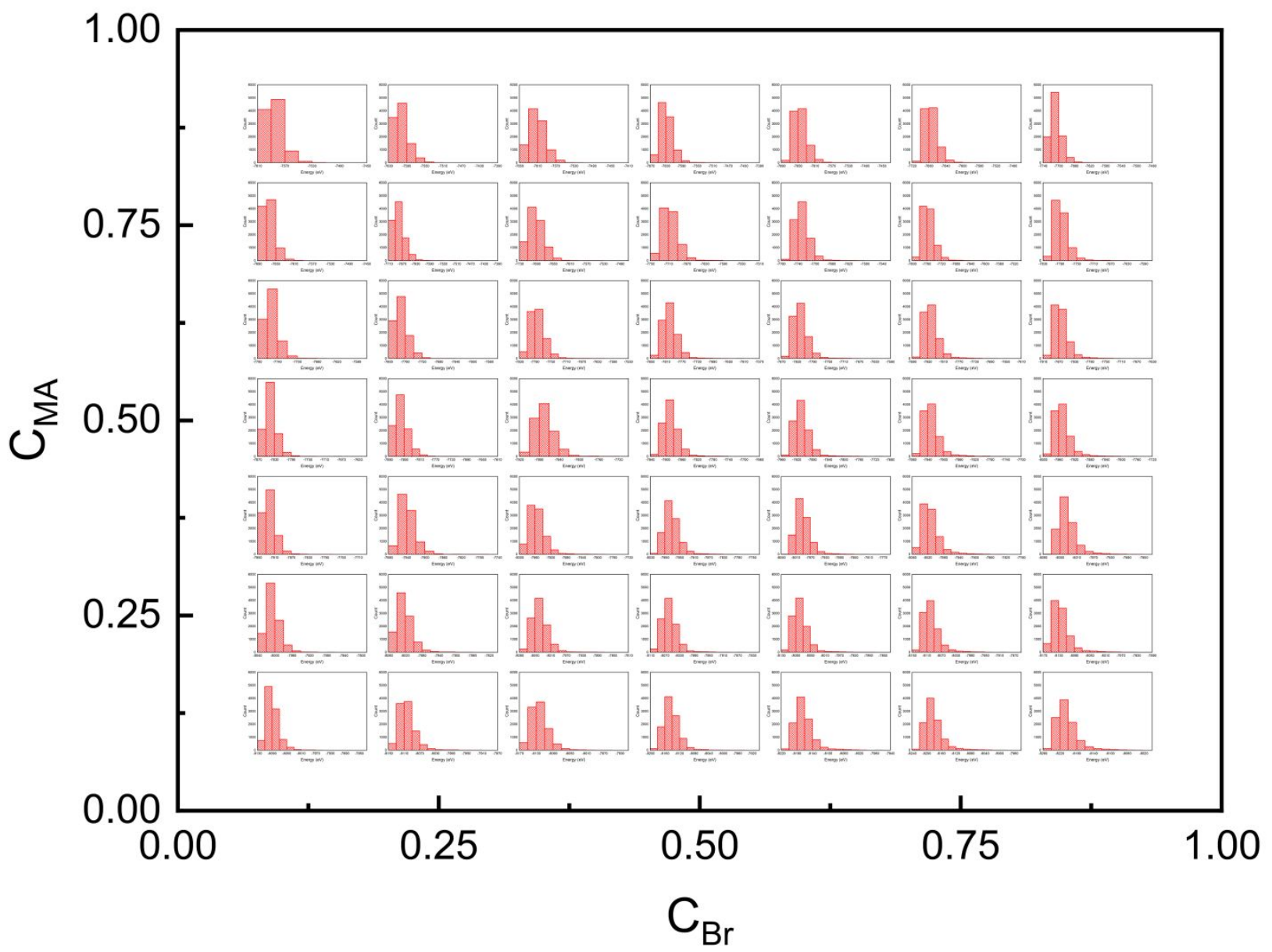

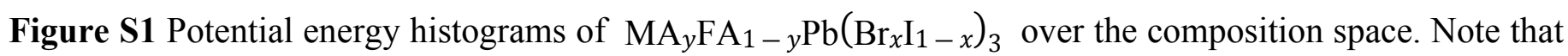
the histograms of compositions with $x, y=0$ or 1 were not shown for clarity. 


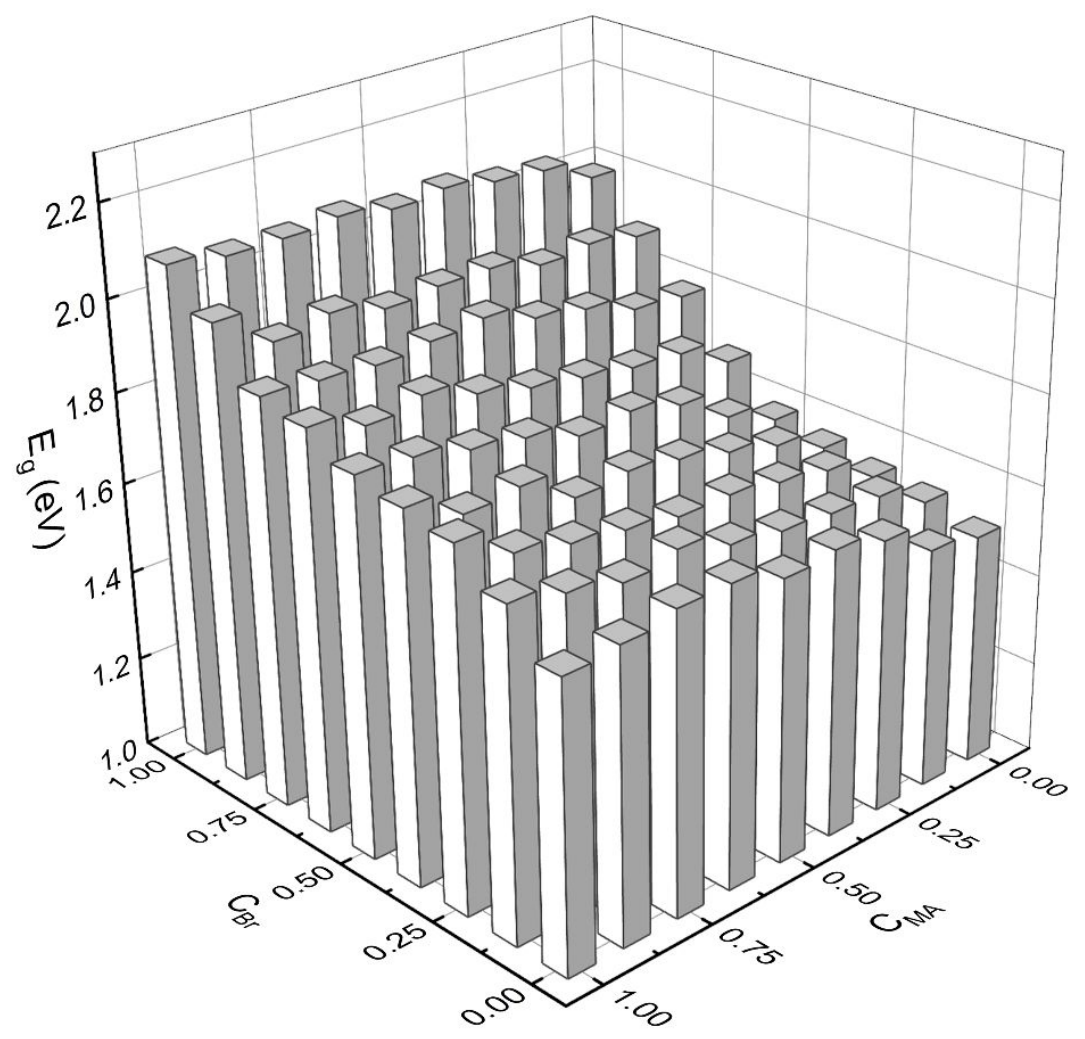

Figure S2 Calculated band gap energies of $\mathrm{MA}_{y} \mathrm{FA}{ }_{1-y} \mathrm{~Pb}\left(\mathrm{Br}_{x} \mathrm{I}_{1-x}\right)_{3}$ over the composition space 

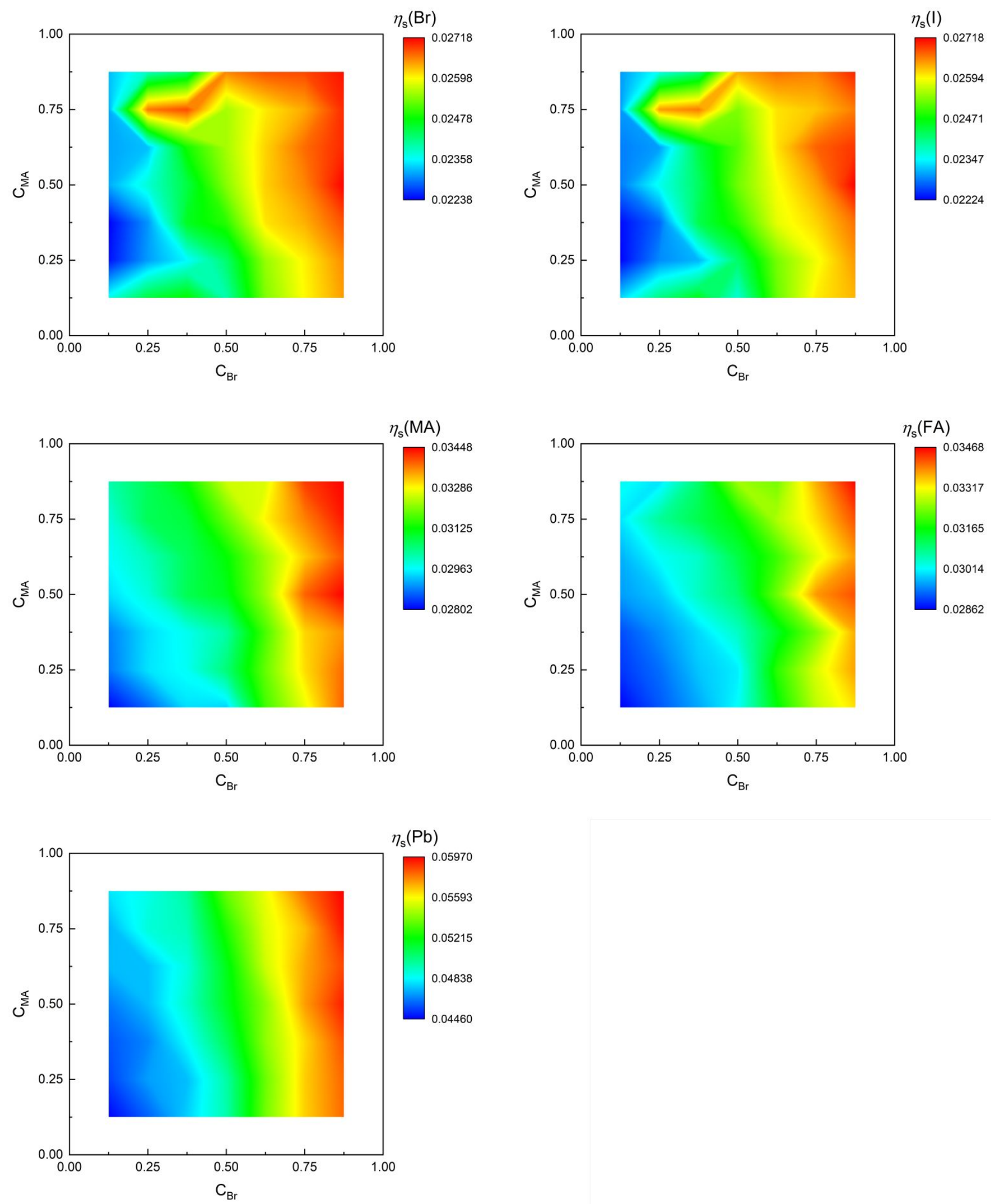

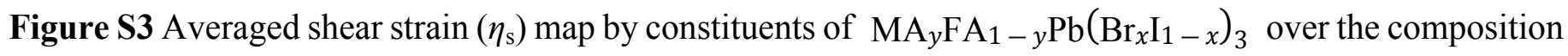
space. 

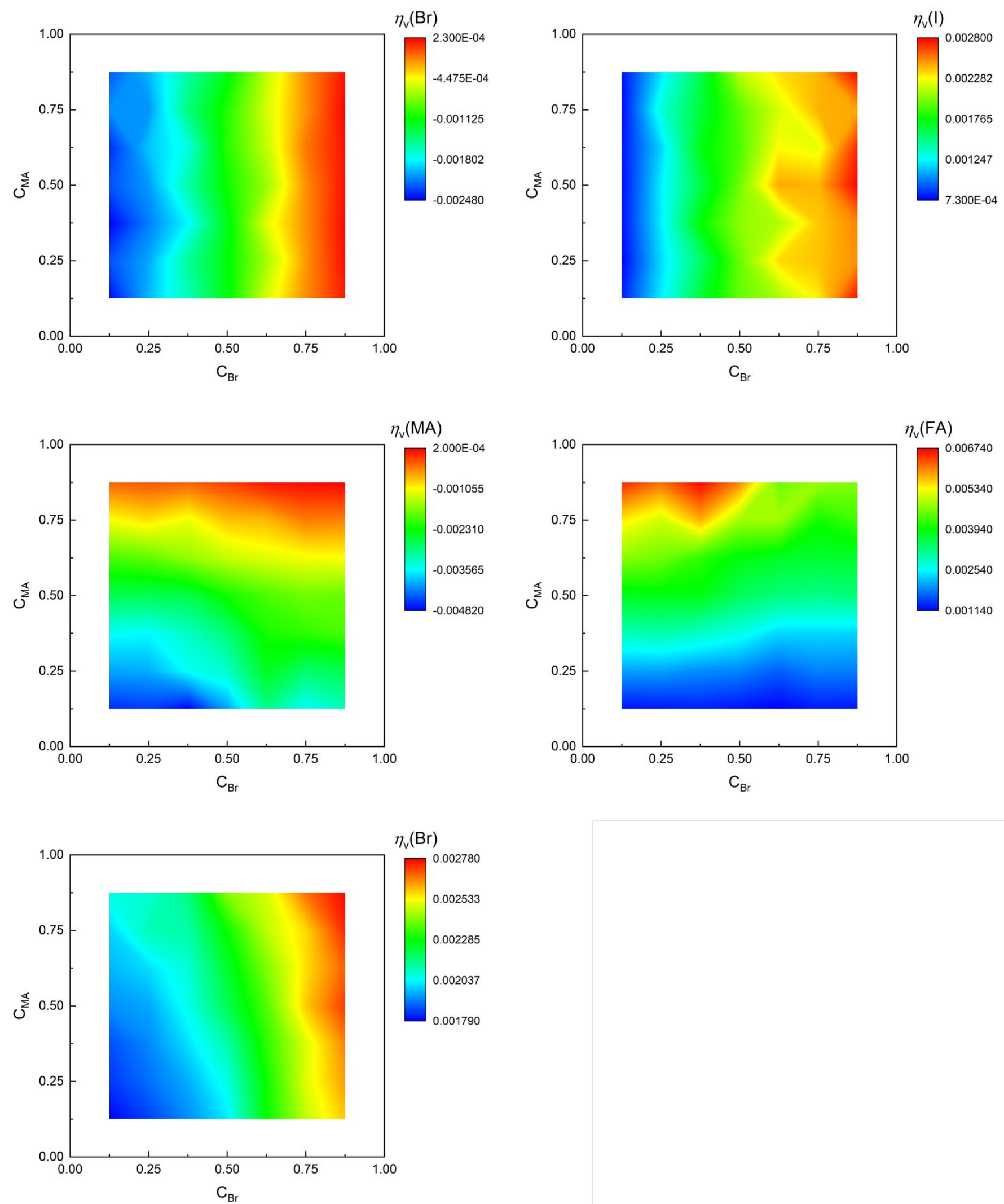

Figure S4 Averaged volumetric strain $\left(\eta_{\mathrm{v}}\right)$ map by constituents of $\mathrm{MA}_{y} \mathrm{FA}_{1-y} \mathrm{~Pb}\left(\mathrm{Br}_{x} \mathrm{I}_{1-x}\right)_{3}$ over the composition space. 
$G^{I I}$

\begin{tabular}{|c|c|c|}
\hline Index & Neighbor Specie & $\eta$ \\
\hline 1 & $\mathrm{Br}$ & 0.05 \\
\hline 2 & $\mathrm{C}$ & 0.05 \\
\hline 3 & $\mathrm{H}$ & 0.05 \\
\hline 4 & I & 0.05 \\
\hline 5 & $\mathrm{~N}$ & 0.05 \\
\hline 6 & $\mathrm{~Pb}$ & 0.05 \\
\hline 7 & $\mathrm{Br}$ & 4 \\
\hline 8 & $\mathrm{C}$ & 4 \\
\hline 9 & $\mathrm{H}$ & 4 \\
\hline 10 & I & 4 \\
\hline 11 & $\mathrm{~N}$ & 4 \\
\hline 12 & $\mathrm{~Pb}$ & 4 \\
\hline 13 & $\mathrm{Br}$ & 20 \\
\hline 14 & $\mathrm{C}$ & 20 \\
\hline 15 & $\mathrm{H}$ & 20 \\
\hline 16 & I & 20 \\
\hline 17 & $\mathrm{~N}$ & 20 \\
\hline 18 & $\mathrm{~Pb}$ & 20 \\
\hline 19 & $\mathrm{Br}$ & 80 \\
\hline 20 & $\mathrm{C}$ & 80 \\
\hline 21 & $\mathrm{H}$ & 80 \\
\hline 22 & I & 80 \\
\hline 23 & $\mathrm{~N}$ & 80 \\
\hline 24 & $\mathrm{~Pb}$ & 80 \\
\hline
\end{tabular}

$G^{I I I}$

\begin{tabular}{|c|c|c|c|c|c|}
\hline Index & Neighbor Specie 2 & Neighbor Specie 3 & $\eta$ & $\lambda$ & $\zeta$ \\
\hline 1 & $\mathrm{Br}$ & $\mathrm{Br}$ & 0.005 & 1 & 1 \\
\hline 2 & $\mathrm{Br}$ & $\mathrm{C}$ & 0.005 & 1 & 1 \\
\hline 3 & $\mathrm{Br}$ & $\mathrm{H}$ & 0.005 & 1 & 1 \\
\hline 4 & $\mathrm{Br}$ & $\mathrm{I}$ & 0.005 & 1 & 1 \\
\hline 5 & $\mathrm{Br}$ & $\mathrm{N}$ & 0.005 & 1 & 1 \\
\hline 6 & $\mathrm{Br}$ & $\mathrm{Pb}$ & 0.005 & 1 & 1 \\
\hline 7 & $\mathrm{C}$ & $\mathrm{C}$ & 0.005 & 1 & 1 \\
\hline 8 & $\mathrm{C}$ & $\mathrm{H}$ & 0.005 & 1 & 1 \\
\hline 9 & $\mathrm{C}$ & $\mathrm{I}$ & 0.005 & 1 & 1 \\
\hline 10 & $\mathrm{C}$ & $\mathrm{N}$ & 0.005 & 1 & 1 \\
\hline 11 & $\mathrm{C}$ & $\mathrm{Pb}$ & 0.005 & 1 & 1 \\
\hline 12 & $\mathrm{H}$ & $\mathrm{H}$ & & \\
\hline
\end{tabular}




\begin{tabular}{|c|c|c|c|c|c|}
\hline 13 & $\mathrm{H}$ & I & 0.005 & 1 & 1 \\
\hline 14 & $\mathrm{H}$ & $\mathrm{N}$ & 0.005 & 1 & 1 \\
\hline 15 & $\mathrm{H}$ & $\mathrm{Pb}$ & 0.005 & 1 & 1 \\
\hline 16 & I & I & 0.005 & 1 & 1 \\
\hline 17 & I & $\mathrm{N}$ & 0.005 & 1 & 1 \\
\hline 18 & I & $\mathrm{Pb}$ & 0.005 & 1 & 1 \\
\hline 19 & $\mathrm{~N}$ & $\mathrm{~N}$ & 0.005 & 1 & 1 \\
\hline 20 & $\mathrm{~N}$ & $\mathrm{~Pb}$ & 0.005 & 1 & 1 \\
\hline 21 & $\mathrm{~Pb}$ & $\mathrm{~Pb}$ & 0.005 & 1 & 1 \\
\hline 22 & $\mathrm{Br}$ & $\mathrm{Br}$ & 0.005 & -1 & 1 \\
\hline 23 & $\mathrm{Br}$ & $\mathrm{C}$ & 0.005 & -1 & 1 \\
\hline 24 & $\mathrm{Br}$ & $\mathrm{H}$ & 0.005 & -1 & 1 \\
\hline 25 & $\mathrm{Br}$ & I & 0.005 & -1 & 1 \\
\hline 26 & $\mathrm{Br}$ & $\mathrm{N}$ & 0.005 & -1 & 1 \\
\hline 27 & $\mathrm{Br}$ & $\mathrm{Pb}$ & 0.005 & -1 & 1 \\
\hline 28 & $\mathrm{C}$ & $\mathrm{C}$ & 0.005 & -1 & 1 \\
\hline 29 & $\mathrm{C}$ & $\mathrm{H}$ & 0.005 & -1 & 1 \\
\hline 30 & $\mathrm{C}$ & I & 0.005 & -1 & 1 \\
\hline 31 & $\mathrm{C}$ & $\mathrm{N}$ & 0.005 & -1 & 1 \\
\hline 32 & $\mathrm{C}$ & $\mathrm{Pb}$ & 0.005 & -1 & 1 \\
\hline 33 & $\mathrm{H}$ & $\mathrm{H}$ & 0.005 & -1 & 1 \\
\hline 34 & $\mathrm{H}$ & I & 0.005 & -1 & 1 \\
\hline 35 & $\mathrm{H}$ & $\mathrm{N}$ & 0.005 & -1 & 1 \\
\hline 36 & $\mathrm{H}$ & $\mathrm{Pb}$ & 0.005 & -1 & 1 \\
\hline 37 & I & I & 0.005 & -1 & 1 \\
\hline 38 & I & $\mathrm{N}$ & 0.005 & -1 & 1 \\
\hline 39 & I & $\mathrm{Pb}$ & 0.005 & -1 & 1 \\
\hline 40 & $\mathrm{~N}$ & $\mathrm{~N}$ & 0.005 & -1 & 1 \\
\hline 41 & $\mathrm{~N}$ & $\mathrm{~Pb}$ & 0.005 & -1 & 1 \\
\hline 42 & $\mathrm{~Pb}$ & $\mathrm{~Pb}$ & 0.005 & -1 & 1 \\
\hline 43 & $\mathrm{Br}$ & $\mathrm{Br}$ & 0.005 & 1 & 4 \\
\hline 44 & $\mathrm{Br}$ & $\mathrm{C}$ & 0.005 & 1 & 4 \\
\hline 45 & $\mathrm{Br}$ & $\mathrm{H}$ & 0.005 & 1 & 4 \\
\hline 46 & $\mathrm{Br}$ & I & 0.005 & 1 & 4 \\
\hline 47 & $\mathrm{Br}$ & $\mathrm{N}$ & 0.005 & 1 & 4 \\
\hline 48 & $\mathrm{Br}$ & $\mathrm{Pb}$ & 0.005 & 1 & 4 \\
\hline 49 & $\mathrm{C}$ & $\mathrm{C}$ & 0.005 & 1 & 4 \\
\hline 50 & $\mathrm{C}$ & $\mathrm{H}$ & 0.005 & 1 & 4 \\
\hline 51 & $\mathrm{C}$ & $\mathrm{I}$ & 0.005 & 1 & 4 \\
\hline 52 & $\mathrm{C}$ & $\mathrm{N}$ & 0.005 & 1 & 4 \\
\hline 53 & $\mathrm{C}$ & $\mathrm{Pb}$ & 0.005 & 1 & 4 \\
\hline
\end{tabular}




\begin{tabular}{|c|c|c|c|c|c|}
\hline 54 & $\mathrm{H}$ & $\mathrm{H}$ & 0.005 & 1 & 4 \\
\hline 55 & $\mathrm{H}$ & I & 0.005 & 1 & 4 \\
\hline 56 & $\mathrm{H}$ & $\mathrm{N}$ & 0.005 & 1 & 4 \\
\hline 57 & $\mathrm{H}$ & $\mathrm{Pb}$ & 0.005 & 1 & 4 \\
\hline 58 & I & I & 0.005 & 1 & 4 \\
\hline 59 & I & $\mathrm{N}$ & 0.005 & 1 & 4 \\
\hline 60 & I & $\mathrm{Pb}$ & 0.005 & 1 & 4 \\
\hline 61 & $\mathrm{~N}$ & $\mathrm{~N}$ & 0.005 & 1 & 4 \\
\hline 62 & $\mathrm{~N}$ & $\mathrm{~Pb}$ & 0.005 & 1 & 4 \\
\hline 63 & $\mathrm{~Pb}$ & $\mathrm{~Pb}$ & 0.005 & 1 & 4 \\
\hline 64 & $\mathrm{Br}$ & $\mathrm{Br}$ & 0.005 & -1 & 4 \\
\hline 65 & $\mathrm{Br}$ & $\mathrm{C}$ & 0.005 & -1 & 4 \\
\hline 66 & $\mathrm{Br}$ & $\mathrm{H}$ & 0.005 & -1 & 4 \\
\hline 67 & $\mathrm{Br}$ & I & 0.005 & -1 & 4 \\
\hline 68 & $\mathrm{Br}$ & $\mathrm{N}$ & 0.005 & -1 & 4 \\
\hline 69 & $\mathrm{Br}$ & $\mathrm{Pb}$ & 0.005 & -1 & 4 \\
\hline 70 & $\mathrm{C}$ & $\mathrm{C}$ & 0.005 & -1 & 4 \\
\hline 71 & $\mathrm{C}$ & $\mathrm{H}$ & 0.005 & -1 & 4 \\
\hline 72 & $\mathrm{C}$ & I & 0.005 & -1 & 4 \\
\hline 73 & $\mathrm{C}$ & $\mathrm{N}$ & 0.005 & -1 & 4 \\
\hline 74 & $\mathrm{C}$ & $\mathrm{Pb}$ & 0.005 & -1 & 4 \\
\hline 75 & $\mathrm{H}$ & $\mathrm{H}$ & 0.005 & -1 & 4 \\
\hline 76 & $\mathrm{H}$ & I & 0.005 & -1 & 4 \\
\hline 77 & $\mathrm{H}$ & $\mathrm{N}$ & 0.005 & -1 & 4 \\
\hline 78 & $\mathrm{H}$ & $\mathrm{Pb}$ & 0.005 & -1 & 4 \\
\hline 79 & $\mathrm{I}$ & $\mathrm{I}$ & 0.005 & -1 & 4 \\
\hline 80 & I & $\mathrm{N}$ & 0.005 & -1 & 4 \\
\hline 81 & I & $\mathrm{Pb}$ & 0.005 & -1 & 4 \\
\hline 82 & $\mathrm{~N}$ & $\mathrm{~N}$ & 0.005 & -1 & 4 \\
\hline 83 & $\mathrm{~N}$ & $\mathrm{~Pb}$ & 0.005 & -1 & 4 \\
\hline 84 & $\mathrm{~Pb}$ & $\mathrm{~Pb}$ & 0.005 & -1 & 4 \\
\hline
\end{tabular}

Table S1 Parameters of $G^{I I}$ and $G^{I I I}$ descriptors for all elements in this study 


\begin{tabular}{|c|c|c|c|c|c|c|c|c|c|c|}
\hline \multirow{2}{*}{\multicolumn{2}{|c|}{$a_{\text {lat }}(\mathbf{A})$}} & \multicolumn{9}{|c|}{$\mathrm{C}_{\mathrm{MA}}$} \\
\hline & & 0 & 0.125 & 0.25 & 0.375 & 0.5 & 0.625 & 0.75 & 0.875 & 1 \\
\hline \multirow{9}{*}{$\mathrm{C}_{\mathrm{Br}}$} & 0 & 6.345 & 6.310 & 6.275 & 6.237 & 6.207 & 6.169 & 6.135 & 6.092 & 6.060 \\
\hline & 0.125 & 6.339 & 6.307 & 6.269 & 6.235 & 6.199 & 6.168 & 6.130 & 6.098 & 6.060 \\
\hline & 0.25 & 6.333 & 6.297 & 6.265 & 6.231 & 6.195 & 6.162 & 6.127 & 6.094 & 6.058 \\
\hline & 0.375 & 6.327 & 6.294 & 6.258 & 6.224 & 6.188 & 6.158 & 6.124 & 6.089 & 6.057 \\
\hline & 0.5 & 6.322 & 6.283 & 6.252 & 6.219 & 6.186 & 6.152 & 6.119 & 6.084 & 6.054 \\
\hline & 0.625 & 6.315 & 6.277 & 6.248 & 6.216 & 6.183 & 6.149 & 6.113 & 6.083 & 6.052 \\
\hline & 0.75 & 6.308 & 6.273 & 6.242 & 6.211 & 6.182 & 6.145 & 6.111 & 6.082 & 6.050 \\
\hline & 0.875 & 6.299 & 6.266 & 6.232 & 6.208 & 6.173 & 6.140 & 6.107 & 6.080 & 6.048 \\
\hline & 1 & 6.291 & 6.262 & 6.228 & 6.204 & 6.167 & 6.135 & 6.103 & 6.075 & 6.046 \\
\hline
\end{tabular}

Table S2 The lattice parameters of $\mathrm{MA}_{y} \mathrm{FA}_{1-y} \mathrm{~Pb}\left(\mathrm{Br}_{x} \mathrm{I}_{1-x}\right)_{3}$ over the composition space for Monte Carlo sampling. 


\begin{tabular}{|c|c|c|c|c|c|c|c|c|c|c|}
\hline \multirow{2}{*}{\multicolumn{2}{|c|}{$E_{g}(e V)$}} & \multicolumn{9}{|c|}{$\mathbf{C}_{\mathrm{MA}}$} \\
\hline & & 0 & 0.125 & 0.25 & 0.375 & 0.5 & 0.625 & 0.75 & 0.875 & 1 \\
\hline \multirow{9}{*}{$\mathrm{C}_{\mathrm{Br}}$} & 0 & 1.51 & 1.53 & 1.60 & 1.63 & 1.62 & 1.66 & 1.66 & 1.64 & 1.63 \\
\hline & 0.125 & 1.54 & 1.60 & 1.61 & 1.62 & 1.64 & 1.68 & 1.66 & 1.69 & 1.72 \\
\hline & 0.25 & 1.55 & 1.61 & 1.63 & 1.65 & 1.65 & 1.67 & 1.69 & 1.72 & 1.79 \\
\hline & 0.375 & 1.57 & 1.62 & 1.65 & 1.68 & 1.70 & 1.69 & 1.76 & 1.75 & 1.81 \\
\hline & 0.5 & 1.59 & 1.64 & 1.71 & 1.74 & 1.73 & 1.77 & 1.79 & 1.82 & 1.83 \\
\hline & 0.625 & 1.68 & 1.74 & 1.75 & 1.77 & 1.79 & 1.82 & 1.86 & 1.84 & 1.88 \\
\hline & 0.75 & 1.79 & 1.80 & 1.84 & 1.86 & 1.90 & 1.89 & 1.88 & 1.89 & 1.90 \\
\hline & 0.875 & 1.89 & 1.91 & 1.90 & 1.93 & 1.93 & 1.92 & 1.95 & 1.93 & 2.01 \\
\hline & 1 & 1.99 & 2.04 & 2.05 & 2.07 & 2.06 & 2.08 & 2.07 & 2.07 & 2.09 \\
\hline
\end{tabular}

Table S3 The band gap energies of $\left.\mathrm{MA}_{y} \mathrm{FA}_{1-y} \mathrm{~Pb}_{\left(\mathrm{Br}_{x} \mathrm{I}_{1}-x\right.}\right)_{3}$ over the composition space for Monte Carlo sampling. 